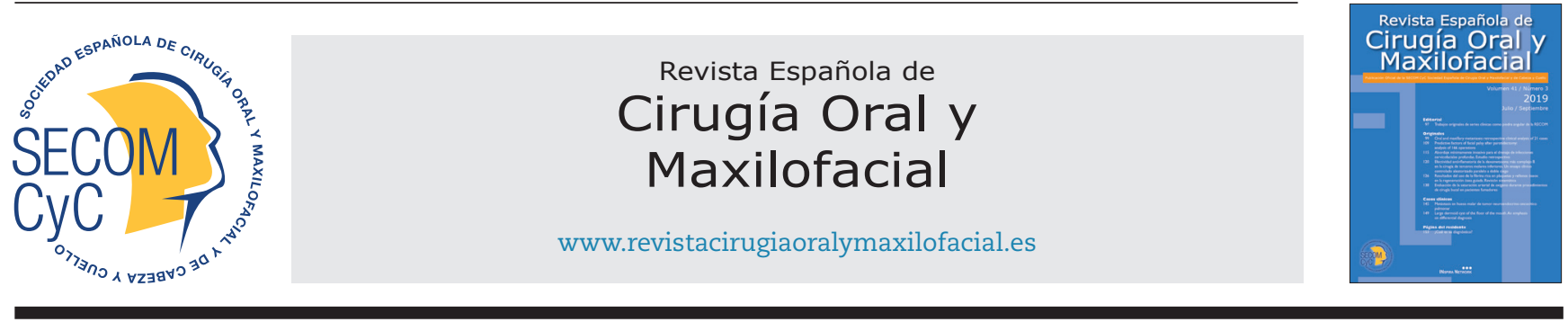

\title{
Original
}

\section{Abordaje mínimamente invasivo para el drenaje de infecciones cervicofaciales profundas. Estudio retrospectivo}

\author{
Nicolás Solano ${ }^{a}$, Luis Sarmiento ${ }^{b}$, Jondalys Lópezc ${ }^{c}$ Manuel Linares ${ }^{d}$, Salomón Ramos \\ y Luis Herreraf
}

${ }^{a}$ Cirujano oral y maxilofacial, Jefe de la Unidad de Cirugía Bucal y Maxilofacial, Servicio de Odontología, Hospital Universitario de Maracaibo, Venezuela. Adjunto de la Unidad de Cabeza y Cuello, Servicio de Cirugía Oncológica, Hospital Universitario de Maracaibo, Venezuela. Profesor Agregado, Postgrado de Cirugía Bucal, Facultad de Odontología, Universidad del Zulia, Venezuela. ${ }^{\text {CCirujano }}$ bucal, Profesor Asociado, Posgrado de Cirugía Bucal, Facultad de Odontología, Universidad del Zulia, Venezuela. Adjunto de la Unidad de Cirugía Bucal y Maxilofacial, Servicio de Odontología, Hospital Universitario de Maracaibo, Venezuela. 'Odontólogo, Residente, Posgrado de Cirugía Bucal, Facultad de Odontología, Universidad del Zulia, Venezuela. 'Odontólogo, Residente, Posgrado de Cirugía Bucal, Facultad de Odontología, Universidad del Zulia, Venezuela. eOdontólogo, Residente, Posgrado de Cirugía Bucal, Facultad de Odontología, Universidad del Zulia, Venezuela. ${ }^{\mathrm{O}}$ Odontólogo, MSc en Medicina Bucal, Área de Clínica y Patología, Instituto de Investigaciones, Facultad de Odontología, Universidad del Zulia, Venezuela.

\section{INFORMACIÓN DEL ARTÍCULO}

Historia del artículo:

Recibido el 10 de enero de 2019

Aceptado el 8 de abril de 2019

Palabras clave:

Infecciones cervicales profundas, incisiones, origen dental, polimicrobiano.

\section{R E S U M E N}

Objetivos: La mayoría de las infecciones cervicales profundas son polimicrobianas y de origen dental, y es necesario un tratamiento apropiado y oportuno. El propósito de esta investigación es describir la eficacia de las incisiones mínimamente invasivas para el drenaje de infecciones cervicales profundas.

Materiales y métodos: Estudio descriptivo, retrospectivo y longitudinal en pacientes con infecciones cervicales profundas, que requirieron ingreso hospitalario, tratados quirúrgicamente con múltiples incisiones mínimamente invasivas. Los datos recopilados incluyeron epidemiología, etiología, órganos dentales y espacio cervicofacial involucrado, tamaño de los abscesos, cantidad de material obtenido, tiempo de hospitalización, tiempo de evolución antes del drenaje, técnica anestésica, método de intubación, método quirúrgico y complicaciones.

Resultados: Fueron incluidos un total de 88 pacientes, con un promedio de edad de $39 \pm 14,05$ años y una mayor prevalencia del género femenino. El factor etiológico principal fueron los focos sépticos dentales. El espacio más afectado fue el submandibular, en un $39,2 \%$. El tiempo de evolución antes del drenaje varió entre 1 y 8 días. El promedio del tamaño de los abscesos fue de $6,47 \pm 2,34,4,03 \pm 1,64,3,71 \pm 1,59 \mathrm{~cm}$. Un 88,6\% de los pacientes fueron intervenidos bajo anestesia local y un 11,8\% bajo anestesia general. El promedio de la cantidad de material obtenido fue de 104,41 9 cc. El tiempo promedio de hospitalización fue de $9,43 \pm 3,89$ días. No hubo complicaciones locales ni sistémicas.

Conclusiones: Las incisiones mínimamente invasivas constituyen una alternativa eficaz, segura y con escasas morbilidades para el drenaje de infecciones cervicales profundas.

\footnotetext{
${ }^{*}$ Autor para correspondencia:

Correo electrónico: jondalyslopez@gmail.com (Jondalys López).
}

DOI: 10.20986/recom.2019.1036/2019

1130-0558/@ 2019 SECOM. Publicado por Inspira Network. Este es un artículo Open Access bajo la licencia CC BY-NC-ND (http:// creativecommons.org/licenses/by-nc-nd/4.0/). 


\section{Minimally invasive approach to drainage of deep cervicofacial infections. Retrospective study}

\section{A B S T R A C T}

Keywords:

Deep neck infections, incisions, dental origin, polymicrobial.
Aim: Most of deep cervical infections are polymicrobial and come from a dental origin, and an appropriate treatment is necessary. The present paper aims to describe the efficiency of minimally invasive incisions for the drainage of deep cervical infections.

Materials and methods: A descriptive, retrospective and longitudinal study in patients with deep cervical infections, who required hospital admission. Patients were surgically treated with multiple minimally invasive incisions. Collected data included: epidemiology, etiology, teeth and cevicofacial spaces involved, size of drained abscesses, amount of material obtained, time of total patient admission, time of evolution before drainage anaesthetic technique, intubation method, surgical method and complications.

Results: A total of 88 patients were included in this study, with an average age of $39 \pm 14.05$ years and a higher prevalence in women. Teeth infections were the etiological factor in $100 \%$ of the sample. The most affected space was the submandibular with a $39.2 \%$. The evolution time before drainage varied between 1-8 days. The mean abscess size was $6.47 \pm 2.34$, $4.03 \pm 1.64,3.71 \pm 1.59 \mathrm{~cm}$. In terms of anaesthetic techniques, $88.6 \%$ patients were treated under local anesthesia and $11.3 \%$ patients were treated under general anesthesia. The average of the amount of material obtained was $104.41 \pm 9 \mathrm{cc}$. The average hospitalization time was $9.43 \pm 3.89$ days. There were no systemic or local complications.

Conclusions: Minimally invasive incisions represent an effective and safe alternative, with low morbidity, for the drainage of deep cervical infections.

\section{INTRODUCCIÓN}

La mayoría de las infecciones cervicales tienen su origen en focos sépticos dentales ${ }^{1}$. Para el cirujano oral y maxilofacial es muy importante conocer el manejo médico-quirúrgico de esta enfermedad que involucra los espacios profundos del cuello y, asimismo, decidir qué terapia antimicrobiana utilizar, manejar el soporte nutricional y de fluidos, conocer cuándo operar y, no menos importante, cómo asegurar la vía aérea. Para poder tomar estas decisiones se hace menester conocer y entender las características anatómicas de la región, la etiología de la infección, un protocolo apropiado de diagnóstico y, también, el manejo médico-quirúrgico ${ }^{2}$. El tratamiento de los abscesos profundos del cuello no ha cambiado sustancialmente en las últimas décadas. Los drenajes quirúrgicos abiertos, incluyendo amplias cervicotomías, son aún parte del protocolo de manejo. Estos procedimientos, por lo regular, precisan anestesia general, cuidados postoperatorios intermedios o intensivos y cuidados propios del tejido traumatizado ${ }^{3}$. Existen pocas publicaciones sobre abordajes mínimamente invasivos para el drenaje de estos abscesos y hasta ahora no han adquirido popularidad. Por tal motivo, el propósito de esta investigación es describir la eficacia de incisiones mínimamente invasivas para el drenaje de infecciones cervicales profundas.

\section{MATERIALES Y MÉTODOS}

Se realizó un estudio descriptivo, retrospectivo y longitudinal en pacientes con infecciones cervicales profundas que requirieron ingreso hospitalario en el periodo comprendido entre enero 2015 y junio de 2017, tratados quirúrgicamente con múltiples incisiones mínimamente invasivas en la Unidad de Cirugía Oral y Maxilofacial del Hospital Universitario de Maracaibo-Venezuela. Esta investigación fue aprobada por la junta de revisión local de nuestra institución. El protocolo de atención incluyó la evaluación de la vía aérea mediante nasofibroscopia y estudios de imagen iniciales mediante radiografías simples de cuello (posteroanterior y lateral) para, posteriormente, complementarlo con otros métodos auxiliares de diagnóstico (ecograma de piel y partes blandas, radiografía panorámica, tomografía computarizada de cuello contrastada) e inicio de terapia antibiótica empírica endovenosa (betalactámico más inhibidor de betalactamasas y lincosamina/nitroimidazol: ampicilina + sulbactam 1,5 g y clindamicina $600 \mathrm{mg} /$ metronidazol $500 \mathrm{mg}$ cada 8 horas), terapia analgésica y antiinflamatoria (antiinflamatorios no esteroideos y esteroideos: ketoprofeno $100 \mathrm{mg}$ y dexametasona $8 \mathrm{mg}$ ), fluidoterapia y soporte nutricional. Aunado a esto, el tratamiento quirúrgico incluyó incisión, drenaje, toma de cultivo, biopsia y, finalmente, extracción del órgano dental involucrado.

En cuanto a la técnica quirúrgica, bajo anestesia local y, en ocasiones, anestesia general, se realizaron dos o más incisiones cervicales mínimamente invasivas, de aproximadamente $2 \mathrm{~cm}$ de extensión, siguiendo los criterios de Topazian y Goldberg 4 , incidiendo sobre piel sana, en un pliegue cutáneo natural y en zonas de mayor declive (Figura 1). Posteriormente se realizó una disección roma hasta lograr entrar al o a los espacios involucrados, para luego interconectar dichas incisiones (Figura 2). Una vez culminado el proceso de drenaje, se colocaron drenajes pasivos (tubos de polietileno fenestrados) que permitieron, a su vez, poder realizar lavados dos veces al día con solución fisiológica al 0,9 \% (Figura 3), que fueron removidos cuando el gasto disminuyó significativamente. 


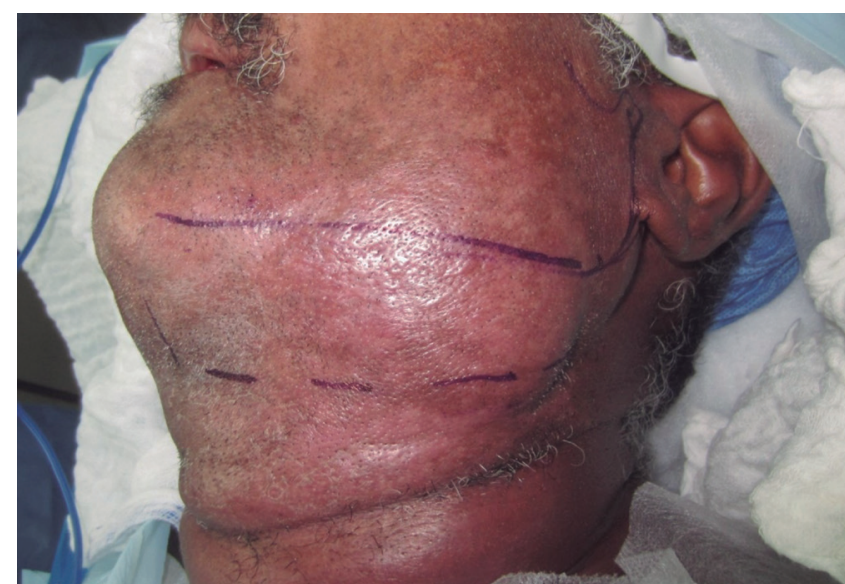

Figura 1. Incisiones cervicales mínimamente invasivas.

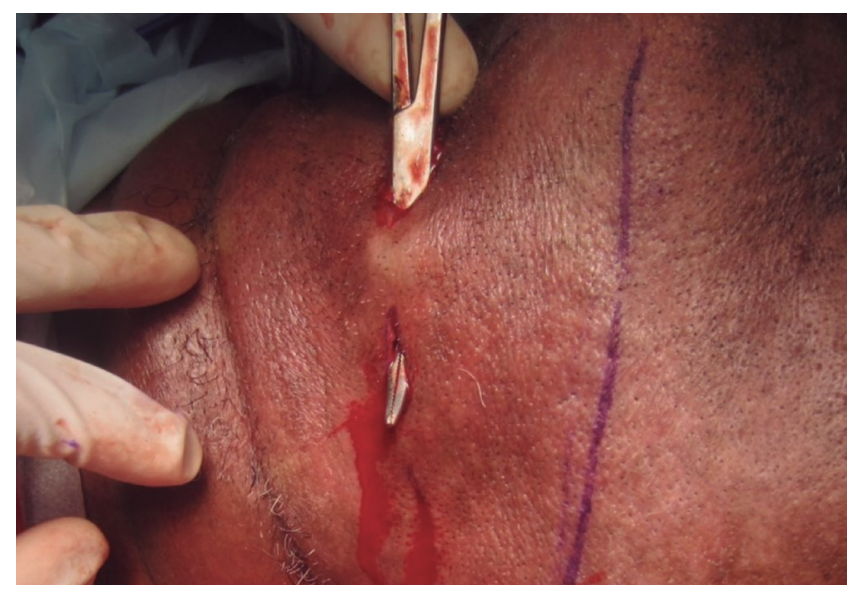

Figura 2. Disección roma de los espacios involucrados.

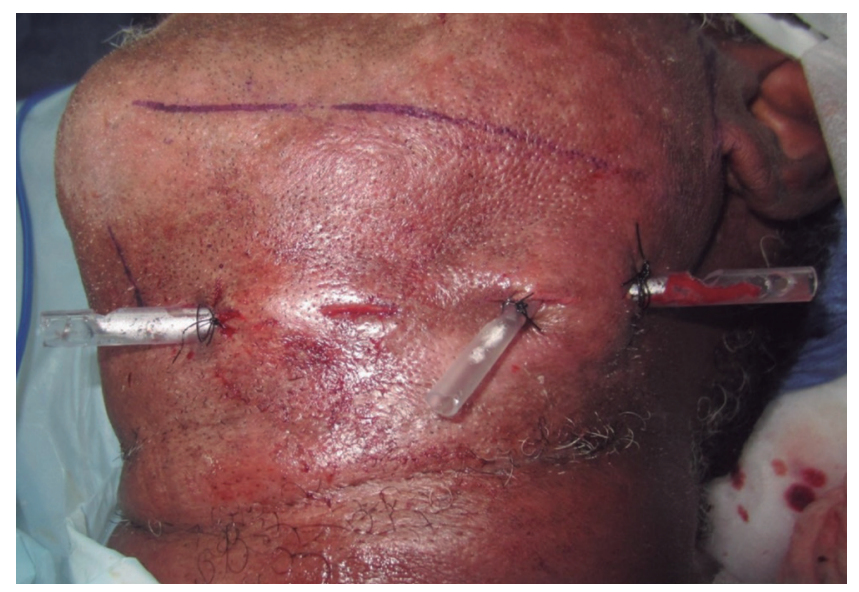

Figura 3. Drenajes pasivos (tubos de polietileno fenestrados).

En este estudio se determinó: epidemiología, etiología, órganos dentales y espacio o espacios cervicofaciales involucrados, tamaño de los abscesos drenados, cantidad de material obtenido, tiempo de ingreso total de los pacientes, tiempo de evolución antes del drenaje, variables anestési- cas que incluyen el tipo de anestesia y el método de intubación (endotraqueal o traqueostomía), método quirúrgico y complicaciones. Para la interpretación de los resultados, los datos se registraron retrospectivamente en formularios de recolección estandarizados. Para el análisis estadístico se creó una base de datos con Microsoft Excel. Las estadísticas descriptivas se calcularon para todas las variables del estudio antes mencionadas.

\section{RESULTADOS}

Un total de 88 pacientes fueron incluidos en este estudio, en edades comprendidas entre 3 y 75 años de edad, con un promedio de $39 \pm 14,05$ años y una mayor prevalencia del género femenino. Los focos sépticos dentales fueron el factor etiológico principal en el $100 \%$ de la muestra, siendo el órgano dental más implicado el tercer molar inferior izquierdo, con un 32,1 \%. El espacio cervical más frecuentemente afectado fue el submandibular, en un 39,2\%. El tiempo de evolución antes del drenaje varió entre 1 y 8 días, con un promedio de 2,88 $\pm 1,95$ días. El promedio del tamaño de los abscesos drenados fue de 6,47 $\pm 2,34$, $4,03 \pm 1,64,3,71 \pm 1,59 \mathrm{~cm}$. En cuanto a las variables anestésicas, 78 pacientes fueron intervenidos bajo anestesia local, lo que supone un 88,6 \%, y 10 bajo anestesia general, es decir, el $11,3 \%$ de los mismos. El método de intubación más frecuente fue el endotraqueal, en un $80 \%$. La cantidad de material obtenido tras el drenaje osciló entre 10 y 378 cc, con un promedio de 104,41 \pm 9 cc. El tiempo de hospitalización de los pacientes varió de 2 a 19 días, con un promedio de 9,43 \pm 3,89 días. Al $100 \%$ de los pacientes se les realizaron incisiones mínimamente invasivas. No hubo complicaciones locales ni sistémicas (Tabla I).

\section{DISCUSIÓN}

Las infecciones cervicales profundas se encuentran dentro de los espacios y los planos fasciales de la región de cabeza y cuello. La mayoría son de origen dental y polimicrobianas, implicando patógenos como Streptococcus viridans, Streptococcus milleri, Prevotella spp., Peptostreptococcus spp. y Klebsiella pneumoniae, razón por la cual la mayoría de los pacientes son tratados empíricamente con regímenes que incluyen clindamicina o betalactámicos, solos o en combinación con metronidazol ${ }^{5,6}$.

Es sumamente necesario un tratamiento apropiado y oportuno de las infecciones cervicales profundas debido a su naturaleza grave y potencialmente mortal, por la capacidad de propagarse a lo largo de los espacios faciales de la cabeza y el cuello, y dar lugar a complicaciones que amenazan la vida ${ }^{5}$. El tratamiento a lo largo de los años se ha basado principalmente en métodos quirúrgicos, soporte nutricional, manejo de las vías respiratorias y una comprensión de la flora microbiológica de estas infecciones ${ }^{2,5-8}$.

La incisión, el drenaje quirúrgico oportuno y la eliminación de los órganos dentales involucrados, además de la colocación de drenajes quirúrgicos ${ }^{8}$, conducirán a una resolución más temprana y permitirá cambiar el entorno local a uno más favorable para el suministro de antibióticos y para 
Tabla I. Distribución de las variables de estudio

\begin{tabular}{lcccc} 
Variable & $\begin{array}{c}\text { Promedio }+ \\
\mathrm{DE}\end{array}$ & Rango & $\begin{array}{c}\mathrm{n} \\
\text { (casos) }\end{array}$ & $\begin{array}{c}\% \\
\text { (casos) }\end{array}$ \\
\hline Edad & $31,71 \pm 14,04$ & $3-75$ & &
\end{tabular}

Género

Masculino

$42 \quad 47,7$

Femenino

$46 \quad 52,3$

Tiempo de evolución

(días)

Número de espacios

Espacio cervicofacial involucrado

Sublingual

Submandibular

Submentoniano

Parafaríngeo

Bucal

Masticatorio

Órganos dentales

Tercer molar inferior

derecho

Segundo molar

inferior derecho

Primer molar

inferior derecho

Segundo premolar

inferior derecho

Primer molar

inferior izquierdo

Segundo molar

inferior izquierdo

Tercer molar inferior

izquierdo

Incisivo lateral

inferior izquierdo

Incisivo central

inferior izquierdo

Incisivo lateral

inferior derecho

Tamaño

Técnica anestésica

Local

General

Material obtenido

Días de

hospitalización

Método de Intubación

Endotraqueal

Traqueostomía

Método quirúrgico

Incisiones

mínimamente

invasivas

Cervicotomías

Complicaciones

Locales

Sí

No

Sistémicas

Sí

No
$2,88 \pm 1,95 \quad 1-8$

$2,48 \pm 0,72 \quad 1-5$

$28 \quad 13,2$

$83 \quad 39,2$

$57 \quad 26,9$

$30 \quad 14,2$

$6 \quad 2,8$

89,1

$25 \quad 23,6$

$2 \quad 1,9$

$5 \quad 4,7$

$1 \quad 0,9$

$30 \quad 28,3$

$6 \quad 5,7$

$34 \quad 32,1$

$1 \quad 0,9$

$1 \quad 0,9$

$1 \quad 0,9$

$6,47 \pm 2,34,4,03 \pm 1,64,3,71 \pm 1,59$

78

88,6

$10 \quad 11,3$

$9,43 \pm 3,89 \quad 2-19$

8

80

220

88

100

$0 \quad 0$

$0 \quad 0$

$88 \quad 100$

$0 \quad 0$

$88 \quad 100$

$104,41 \pm 9 \quad 10-378$ promover la actividad de los mecanismos de defensa del huésped?.

En una investigación realizada por Mücke y cols. ${ }^{9}$ se evaluó el resultado del uso de incisiones intraorales adicionales en comparación con el tratamiento estándar de drenaje extraoral en pacientes con abscesos maxilofaciales odontogénicos. Se incluyeron 205 pacientes, de los cuales 113 fueron hombres $(55,1 \%)$ y 92 mujeres (44,9\%), con una edad media de 47,5 $\pm 18,1$ años (rango 6-90 años), lo que contrasta con nuestros hallazgos, donde los pacientes que cursaban con infecciones cervicales profundas presentaron un rango etario y un promedio menor en comparación con el citado trabajo, y la prevalencia era mayor en el género femenino.

Diversos autores ${ }^{10,11}$ han reportado el origen dental como la causa más común en las infecciones cervicofaciales. Jansisyanont y cols..$^{12}$, en un estudio retrospectivo realizado en 112 pacientes (65 varones y 47 mujeres con edades promedio de 33,35 \pm 19,07 y 33,21 $\pm 20,71$ años, respectivamente) identificaron los factores predisponentes relacionados con los resultados del tratamiento de las infecciones de los espacios maxilofaciales, y la mayoría de las infecciones ocurrieron en pacientes del grupo de 21-40 años. Las infecciones odontogénicas se desarrollaron en su mayoría a partir de los dientes mandibulares posteriores (56 casos, 67,5\%). En 25 de estos 56 casos estaban involucrados terceros molares mandibulares, con una relación órganos dentales \# 3,8-4,8 de 2,57:1, coincidiendo con lo encontrado en el presente estudio.

En este estudio, los espacios cervicofaciales más frecuentemente afectados fueron, en orden descendente, submandibular, submentoniano, parafaríngeo y sublingual, lo que contrasta con lo encontrado por Flynn y cols. ${ }^{13}$, que refirieron que el espacio masticador medial o pterigomandibular es más habitual en las infecciones odontogénicas, siendo el segundo espacio más común el submandibular. Sin embargo, nuestros hallazgos concuerdan con el estudio de Bouloux y cols. ${ }^{8}$, donde el espacio cervicofacial más común fue el submandibular, que estuvo implicado en el 72 \% de los sujetos. La participación del espacio masticador medial o pterigomandibular puede ocurrir secundariamente a la participación de terceros molares inferiores o como resultado de la propagación desde el espacio submandibular. Por lo tanto, el tratamiento precoz y agresivo de la infección en el espacio submandibular puede prevenir la extensión al espacio masticador medial y explicar la menor frecuencia de afectación del mismo encontrada en este estudio, además de reducir la propagación de la infección al resto de los espacios cervicales próximos.

El manejo no quirúrgico o la intervención mínimamente invasiva ${ }^{5}$ o guiada por imágenes (tomografía computarizada o ultrasonido) para la aspiración y el drenaje de infecciones cervicales profundas constituyen una alternativa; sin embargo, estas opciones no se han popularizado y aún son objeto de debate en la bibliografía ${ }^{3}$. Bross-Soriano y cols. ${ }^{10}$ realizaron una revisión retrospectiva de 121 pacientes con angina de Ludwig tratados quirúrgicamente con pequeñas incisiones. En esta serie, 33 pacientes tuvieron complicaciones importantes como mediastinitis, sepsis o muerte. Sin embargo, se concluyó que el drenaje con pequeñas incisiones era seguro y eficaz en pacientes con angina de Ludwig. Esto coincide con lo encontrado en nuestro estudio, en el cual se realizaron incisiones mínimamente invasivas (> 2) con una técnica quirúrgica similar 
para el drenaje de los espacios cervicofaciales involucrados, sin embargo difiere en que en el presente estudio no se produjeron complicaciones locales y sistémicas.

Gholami y cols. ${ }^{14}$ realizaron un estudio en pacientes con infecciones odontogénicas cuyo tratamiento consistió en antibióticos endovenosos, incisión quirúrgica, drenaje y extracción de los dientes involucrados, describiendo los datos clínicos y sociodemográficos de los mismos. Durante la incisión y el drenaje se utilizó anestesia local y sedoanalgesia en 11 (10,8\%) de los sujetos, y anestesia general en el resto de los casos $(89,2 \%)$, de los cuales en un 85,3\% se empleó intubación endotraqueal por laringoscopia directa, mientras que el 3,9 \% requirieron traqueotomía urgente, lo que difiere de lo ocurrido en nuestro estudio, donde el $88,6 \%$ de los pacientes fueron intervenidos bajo anestesia local y un 11,3\% bajo anestesia general, siendo el método de intubación más frecuente el endotraqueal, en un $80 \%$. Los casos intervenidos con anestesia local están relacionados con infecciones cervicales profundas mayormente asociadas al espacio submandibular, por lo que la utilización de incisiones mínimamente invasivas que implique a este espacio permite una adecuada resolución del cuadro con escasas morbilidades y sin requerir anestesia general.

Las complicaciones graves de las infecciones odontogénicas no son frecuentes. Sin embargo, cuando ocurren, pueden aumentar la duración de la estancia hospitalaria y amenazar la vida de los pacientes ${ }^{15}$. Los hallazgos encontrados en la bibliografía muestran que la participación de los espacios cervicales profundos (faríngeo lateral y retrofaríngeo) indica una extensión rápida de la infección, siendo potencialmente mortal. Por lo tanto, la disfagia y la disnea debidas a la propagación de infecciones cervicales profundas deben ser apreciadas como un indicador significativo de infección grave. En tal situación, el drenaje rápido y agresivo de los espacios afectados y el establecimiento de una vía aérea segura son cruciales para prevenir la mortalidad ${ }^{14}$. En el presente estudio, a pesar de no existir complicaciones locales ni sistémicas, el tiempo de estancia intrahospitalaria de los pacientes es mayor a lo reportado en la bibliografía ${ }^{13-16}$; esto se debe a que, como criterio de nuestro servicio, todos los pacientes con infecciones cervicales profundas deben cumplir aproximadamente 10 días de hospitalización para garantizar una administración adecuada de la terapia antibiótica endovenosa, así como monitorizar la vía aérea y asegurar la eliminación del órgano dental asociado a la enfermedad.

\section{CONCLUSIÓN}

Las incisiones mínimamente invasivas constituyen una alternativa eficaz, segura y con escasas morbilidades para el drenaje de infecciones cervicales profundas de origen dental cuando estas involucran principalmente el espacio submandibular, submentoniano y sublingual, ya que la naturaleza y localización de estos espacios permiten una adecuada evacuación de los abscesos localizados a este nivel, además de brindar la posibilidad de realizar dicho procedimiento quirúrgico bajo anestesia local, evitando la necesidad de los cuidados postoperatorios intermedios o intensivos que conllevan las amplias cervicotomías realizadas con anestesia general.

\section{B I B L I O G R A F Í A}

1. Feldt BA, Webb DE. Neck infections. Atlas Oral Maxillofacial Surg Clin N Am. 2015;23:21-9.

2. Osborn TM, Assael LA, Bell RB. Deep space neck infection: Principles of surgical management. Oral Maxillofacial Sure Clin N Am 2008;20(3):353-65. DOI: 10.1016/j.coms.2008.04.002.

3. Probst FA, Otto S, Sachse R, Cornelius CP. Minimally-invasive catheter drainage of submandibular abscesses. Br J Oral Maxillofac Surg. 2013;51(7):e199-e200. DOI: 10.1016/j.bjoms.2012.06.013.

4. Topazian RG, Goldberg MH. Odontogenic infections and deep fascial space infections of dental origin. En: Topazian RG, editor. Oral and maxillofacial infections. $4^{a}$ ed. Philadelphia: WB Saunders Company; 2002.

5. Shanti RM, Aziz SR. Should we wait for development of an abscess before we perform incision and drainage? Oral Maxillofac Surg Clin North Am. 2011;23(4):513-8. DOI: 10.1016/j. coms.2011.07.004.

6. Caccamese JF Jr, Coletti DP. Deep neck infections: clinical considerations in aggressive disease. Oral Maxillofac Surg Clin North Am. 2008;20(3):367-80. DOI: 10.1016/j.coms.2008.03.001.

7. Govea-Camacho LH, Astudillo-Carrera A, Hermosillo-Sandoval JM, Rodríguez-Reynoso S, González-Ojeda A, Fuentes-Orozco C. Impact of vacuum-assisted closure management in deep neck abscesses. Cir Cir. 2016;84(4):275-81. DOI: 10.1016/j.circir.2015.12.004.

8. Bouloux GF, Wallace J, Xue W. Irrigating drains for severe odontogenic infections do not improve outcome. J Oral Maxillofac Surg. 2013;71(1):42-6.

9. Mücke T, Dujka N, Ermer MA, Wolff KD, Kesting M, Mitchell DA, et al. The value of early intraoral incisions in patients with perimandibular odontogenic maxillofacial abscesses. J Craniomaxillofac Surg. 2015;43(2):220-3. DOI: 10.1016/j.jcms.2014.11.009.

10. Bross-Soriano D, Arrieta-Gómez JR, Prado-Calleros H, Schimelmitz-Idi J, Jorba-Basave S. Management of Ludwig's angina with small neck incisions: 18 years experience. Otolaryngol Head Neck Surg. 2004;130(6):712-7. DOI: 10.1016/j.otohns.2003.09.036.

11. Daramola OO, Flanagan CE, Maisel RH, Odland RM. Diagnosis and treatment of deep neck space abscesses. Otolaryngol Head Neck Surg. 2009;141(1):123-30. DOI: 10.1016/j.otohns.2009.03.033.

12. Jansisyanont $P$, Kasemsai W, Bamroong P. Factors related to the treatment outcome of maxillofacial fascia space infection. J Oral Maxillofac Surg Med Pathol. 2015;27:458-64.

13. Flynn TR, Shanti RM, Levi MH, Adamo AK, Kraut RA, Trieger N. Severe odontogenic infections, part 1: prospective report. J Oral Maxillofac Surg. 2006;64(7):1093-103. DOI: 10.1016/j. joms.2006.03.015.

14. Gholami M, Mohammadi H, Amiri N, Khalife H. Key factors of odontogenic infections requiring hospitalization: A retrospective study of 102 cases. J Oral Maxillofac Surg Med Pathol. 2017;29(5):394-9.

15. Alotaibi N, Cloutier L, Khaldoun E, Bois E, Chirat M, Salvan D. Criteria for admission of odontogenic infections at high risk of deep neck space infection. Eur Ann Otorhinolaryngol Head Neck Dis. 2015;132(5):261-4. DOI: 10.1016/j.anorl.2015.08.007.

16. Flynn TR, Shanti RM, Hayes C. Severe odontogenic infections, part 2: prospective outcomes study. J Oral Maxillofac Surg. 2006;64(7):1104-13. DOI: 10.1016/j.joms.2006.03.031. 